\title{
Multiple Steady States in Thermally Coupled Distillation Sequences: Revisiting the Design, Energy Optimization, and Control
}

\author{
Raúl Delgado-Delgado, ${ }^{\dagger}$ Fabricio Omar Barroso-Muñoz, ${ }^{\dagger}$ Juan Gabriel Segovia-Hernández, $^{\dagger}$ \\ Héctor Hernández-Escoto, ${ }^{\dagger}$ Agustín Jaime Castro-Montoya, ${ }^{\ddagger}$ Vicente Rico-Ramírez, ${ }^{\S}$ \\ and Salvador Hernández ${ }^{* \dagger}$
}
${ }^{\dagger}$ DCNyE, Departamento de Ingeniería Química, Universidad de Guanajuato, Campus Guanajuato, Noria Alta s/n, 36050, Guanajuato, Gto. México
${ }^{\ddagger}$ Facultad de Ingeniería Química, Universidad Michoacana de San Nicolás de Hidalgo, Francisco J. Múgica s/n, Ciudad Universitaria, 58060, Morelia, Michoacán, México
${ }^{\S}$ Departamento de Ingeniería Química, Instituto Tecnológico de Celaya, Av. Tecnólogico y García Cubas S/N, Celaya, Guanajuato 38010, México

\section{Supporting Information}

ABSTRACT: The design, optimization, and control of thermally coupled distillation sequences with side columns have been extensively studied in the literature. Energy savings of up to $30 \%$ have been reported for such systems; further, dynamic simulations show that the theoretical control properties and closed-loop dynamic responses of thermally coupled distillation sequences are better than those of the conventional distillation sequences. In this paper, we have extended the optimization search for the energy required on the separation of a ternary mixture, detecting two feasible solutions for the energy required in the reboiler for the same value of the interconnecting stream (multiple steady states), filling the gap about this topic on these systems. The multiple steady states detected in the thermally coupled distillation sequences (TCDS) were subjected to a controllability analysis and a closed-loop dynamic study. According to the controllability analysis, the steady state with higher demand of energy in the reboiler presented better theoretical control properties than those of the steady state with lower energy requirement in the reboiler. Consistent with the controllability results, when the two steady states were studied under closed-loop using PI controllers, the best dynamic responses for set point tracking and load rejection were exhibited by the steady state design with the higher energy requirement in the reboiler.

\section{INTRODUCTION}

Because distillation is an industrial operation with high energy demand and a very low thermodynamic efficiency, ${ }^{1,2}$ several studies have been conducted in order to mitigate those problems. Tedder and Rudd ${ }^{1}$ presented a very complete study of the energy requirements of eight distillation sequences for the separation of ternary mixtures. They compared complex distillation sequences, including the thermally coupled distillation sequences (TCDS) with side columns, against the classical direct and indirect distillation sequences, obtaining energy savings of around $30 \%$ by using the TCDS with side columns in comparison to conventional distillation sequences for ternary mixtures $(A, B, C)$ with low content of the intermediate component.

Glinos and Malone ${ }^{3}$ estimated the amount of vapor generated in the reboiler under minimum reflux conditions for complex and conventional distillation sequences. They concluded that savings of around $30 \%$ in the minimum vapor generated in the reboilers were achieved by using TCDS with side columns.

Several studies have reported that TCDS can reduce capital costs and energy requirements in the range between $30 \%$ and $50 \%$ in comparison to the conventional distillation sequences. $^{4-7}$
Despite the energy savings achieved in the complex distillation sequences, it was thought that their control and operation could be more difficult than in the conventional distillation sequences due to the presence of recycle streams. However, posterior studies evaluated their control properties $^{8-11}$ and showed that their control properties were as good as those of the conventional distillation sequences and similar closed-loop dynamic responses were presented.

Recently, it has been reported that TCDS and reactive distillation systems can show steady state multiplicity due to their complex physicochemical behavior. ${ }^{12-14}$ In the case of finding multiple steady states, a control properties analysis can help to select those operational parameters that result in the best dynamic performance. In this work, comparative studies are conducted on the theoretical control properties and closedloop dynamics responses for set point tracking and load rejection for some steady states found within the regions of multiplicity in the TDCS systems; then, these studies allow us to select the best steady state in terms of cost and control. This is significant since these kinds of complex distillation sequences

Received: July 9, 2014

Revised: October 20, 2014

Accepted: October 20, 2014

Published: October 20, 2014 
are being implemented in industrial practice using the dividing wall distillation technology. ${ }^{15-20}$

\section{DESIGN AND ENERGY OPTIMIZATION OF THE TCDS WITH SIDE COLUMNS}

The two TCDS systems depicted in Figures 1 and 2 were designed for minimum energy requirements. The direct TCDS

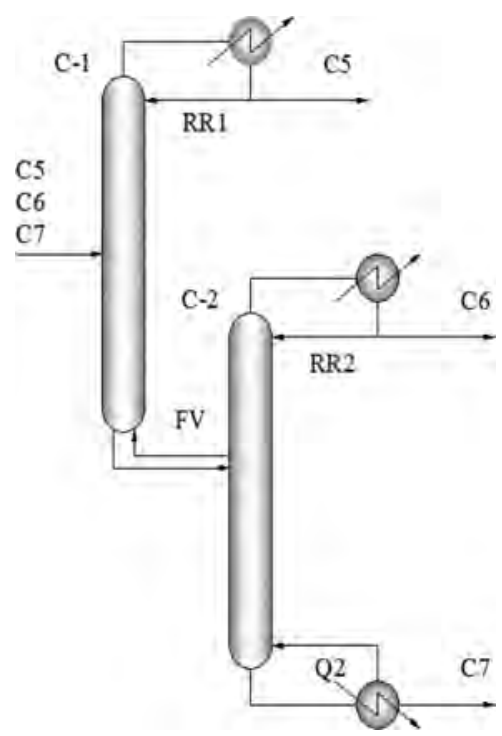

Figure 1. Thermally coupled direct distillation sequence (TCDS-D).

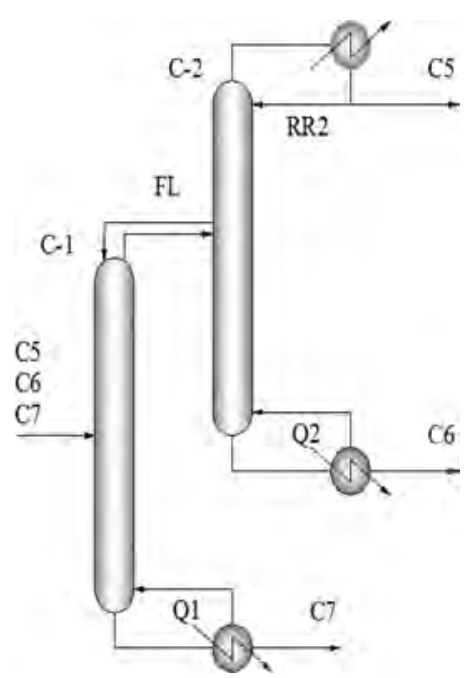

Figure 2. Thermally coupled indirect distillation sequence (TCDS-I).

(TCDS-D) is derived from the conventional direct distillation sequence by replacing the reboiler of the first distillation column (C-1) by a thermal coupling (vapor recycle stream, $\mathrm{FV}$ ). In the case of the indirect TCDS (TCDS-I), the condenser of the first distillation column (C-1) is eliminated due to the liquid recycle stream (FL).

The design method used for both sequences (TCDS-D and TCDS-I) was reported in detail in Hernández and Jiménez ${ }^{4}$ using a dynamic model of an equilibrium stage. The same procedure is applied in this work by using the Aspen Plus process simulator. For the case of TCDS-D, first the conventional distillation sequence was designed by using the short-cut design method of Fenske-Underwood-Gilliland; then, the conventional distillation sequence is simulated by using the rigorous radfrac module of Aspen Plus. The purities of the products were set to 0.98 mole fraction using design specifications. The final design of the TCDS-D is obtained by replacing the reboiler of the first distillation column with a thermal link; the minimum energy requirement in the reboiler Q2 is estimated by varying the vapor recycle stream (FV) for a fixed number of equilibrium stages.

The design of the TCDS-I is obtained in a similar way from the conventional indirect distillation sequence. The indirect distillation sequence is designed by using the same short-cut design method and the design is simulated using the rigorous equilibrium stage contained in the radfrac module with the same purity constraints for the products. The condenser of the first distillation column is then replaced by a liquid recycle stream (FL) that is varied until the minimum energy required in the reboilers $(\mathrm{Q} 1+\mathrm{Q} 2)$ is obtained for a given set of equilibrium stages.

It is important to highlight that the energy required in the reboiler was minimized for a given tray structure in the TCDS configurations, because even for complex distillation columns, the energy supplied to the reboiler is the main component of the total annual cost.

\section{CONTROLLABILITY ANALYSIS}

The theoretical control properties of the TCDS systems were evaluated using the singular value decomposition technique in the frequency domain as used by Hernández and Jiménez. ${ }^{5}$ This technique does not depend on a specific control structure and only open-loop dynamic responses adjusted to linear models are required. The open-loop dynamic responses were obtained in Aspen Dynamics, by implementing a small step change in the input variable (reboiler duty, reflux ratio) and registering the dynamic responses of the product purities. The linear models are grouped into a transfer function matrix in the frequency domain, which is subject to singular value decomposition (SVD):

$$
\mathbf{G}(w j)=\mathbf{U}(w j) \Sigma(w j) \mathbf{V}^{\mathrm{H}}(w j)
$$

where $\mathbf{G}(w j)$ is the transfer matrix function, $\mathbf{U}(w j)$ and $\mathbf{V}(w j)$ are squared matrices and $\Sigma(w j)$ is a diagonal matrix with the same dimensions as $\mathbf{G}(w j)$. There are two important parameters in matrix $\Sigma(w j)$ : the minimum singular value $\left(\sigma_{*}\right)$ and the maximum singular value $\left(\sigma^{*}\right)$. The relationship among them is known as condition number (eq 2).

$$
\gamma^{*}=\frac{\sigma^{*}}{\sigma_{*}}
$$

The minimum singular value is a measure of the invertibility of the system, and therefore represents a measure of the potential problems of the system under feedback control. The condition number reflects the sensitivity of the system under uncertainties in process parameters and modeling errors. These two parameters are important in robust control in order to infer the dynamic behavior under closed-loop operation.

Finally, closed-loop dynamic simulations under feedback control were carried out, and the responses were analyzed to determine the TCDS system with the best dynamic behavior.

\section{CASE STUDY}

The systems studied were (1) TCDS-D depicted in Figure 1 and (2) TCDS-I shown in Figure 2. Both sequences were fed 
with a ternary mixture of $n$-pentane (C5), $n$-hexane (C6), and $n$-heptane (C7) with molar fractions of $0.4,0.2$ and 0.4 , respectively. The purities in the products were set to 0.98 mole fraction in the main component.

\section{RESULTS OF DESIGN AND ENERGY OPTIMIZATION OF THE COMPLEX DISTILLATION SEQUENCES}

The steady state designs of the TCDS schemes are presented in Tables 1 and 2. These designs are obtained using the short cut

Table 1. Important Design Variables of the TCDS-D

\begin{tabular}{lcc}
\multicolumn{1}{c}{ design variable } & column C-1 & column C-2 \\
total stages & 17 & 19 \\
feed stage & 9 & 10 \\
top pressure (psia) & 21.20 & 14.20 \\
bottoms pressure (psia) & 31.20 & 24.70 \\
\hline
\end{tabular}

Table 2. Important Design Variables of the TCDS-I

\begin{tabular}{lcc}
\multicolumn{1}{c}{ design variable } & column C-1 & column C-2 \\
total stages & 21 & 17 \\
feed stage & 11 & 9 \\
top pressure (psia) & 16.50 & 21.20 \\
bottoms pressure (psia) & 26.20 & 31.20 \\
\hline
\end{tabular}

method of Fenske-Underwood-Gilliland, and the final compositions of 0.98 in the products are adjusted using design specifications in the radfrac module of Aspen Plus. These tray structures are fixed during the energy optimization.

Figure 3 shows the results for the minimization of the energy consumption of TCDS-D (Figure 1). Figure $3 \mathrm{a}$ is obtained from a direct search of the minimum energy consumption in the reboiler Q2 of the Column C-2, from low values of the interconnecting vapor stream (FV) to high values of it. The energy demand decreases as FV increases but, for higher values of FV, the energy demand increases again. When the complete search is conducted in the increasing direction of FV (Figure $3 a$, blue curve), the search is continued in the decreasing direction of FV (Figure 3b, green curve), and the heat required in the reboiler is significantly higher. As a result, two very different values of the heat duties are obtained for the same value assigned to FV. For interconnection vapor flows between 127.5 and $145.5 \mathrm{lb}-\mathrm{mol} / \mathrm{h}$, multiple steady states can be observed, and a minimum heat duty of $668.5 \mathrm{~kW}$ is obtained with a value of $134 \mathrm{lb}-\mathrm{mol} / \mathrm{h}$ of FV. Gani and Jorgensen ${ }^{21}$ defined three types of multiplicity for distillation systems: output multiplicity, input multiplicity, and internal state multiplicity. On the basis of the above, the multiplicity of steady states found is an input multiplicity due to the specification of the product purities (outputs).

Figure 4 displays a very similar trend on the search for the energy required in the reboilers of the TCDS-I. Again, a search in the increasing direction of FL provides a complete curve (Figure 4a, blue curve) but, when the search is conducted in the opposite direction, a second curve is obtained with higher values of energy (Figure $4 \mathrm{~b}$, green curve). The TCDS-I also presents a region where two different values of the heat duties can be obtained for the reboiler for a given value of FL. As a result, for TCDS systems is important to obtain the complete search of the minimum energy consumption required in the reboilers since two (very different) solutions can be obtained for the same value of the interconnecting stream. Further, it is
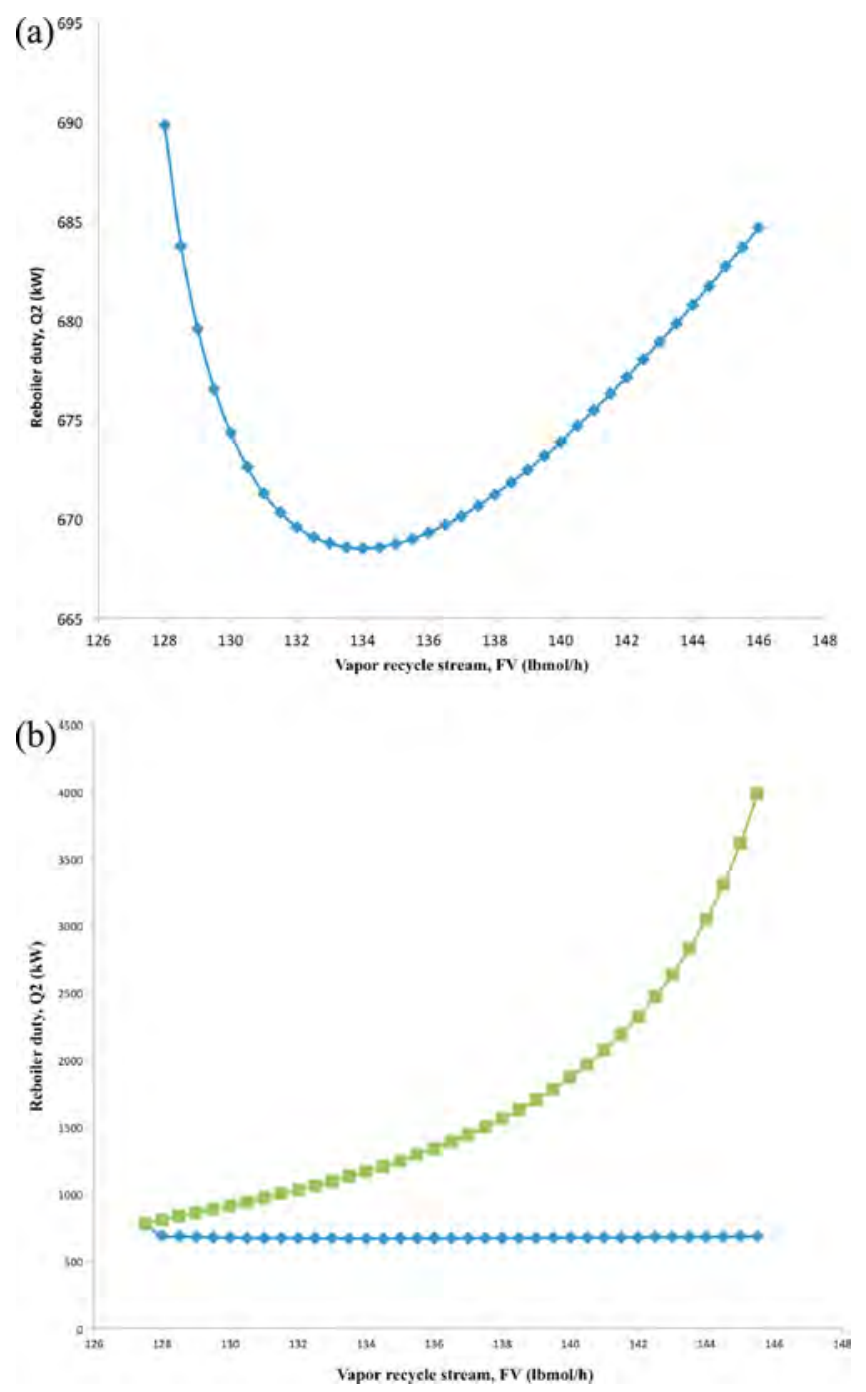

Figure 3. Energy optimization of the TCDS-D: (a) forward search (blue); (b) backward search (green).

important to study the control properties and closed-loop dynamic responses for set point tracking and load rejection in order to detect which steady state is more controllable.

\section{CONTROLLABILITY ANALYSIS RESULTS}

To obtain the control properties, transfer function matrices were determined. For the TCDS-D (see Figure 1), the manipulated variables include the reflux ratio of the first distillation column (RR1) as well as the reflux ratio (RR2) and the reboiler duty (Q2) for the second distillation column. The output variables are the composition of the distillate of the first distillation column ( 55$)$ as well as the composition of the distillate (C6) and the composition of the bottoms product (C7) of the second distillation column. When the TCDS-I is analyzed (see Figure 2), the manipulated variables are Q1, Q2, and RR2, whereas the output variables are C7, C6, and C5. As indicated previously, Aspen Dynamics was used to obtain the dynamic responses of the two steady state designs for a given value of FV or FL depending on the TCDS system under study. Tables 3 and 4 present the design variables for the two states subjected to SVD. The open-loop dynamic responses were adjusted to linear models, and the transfer function matrices can be consulted in the Supporting Information. These 
(a)

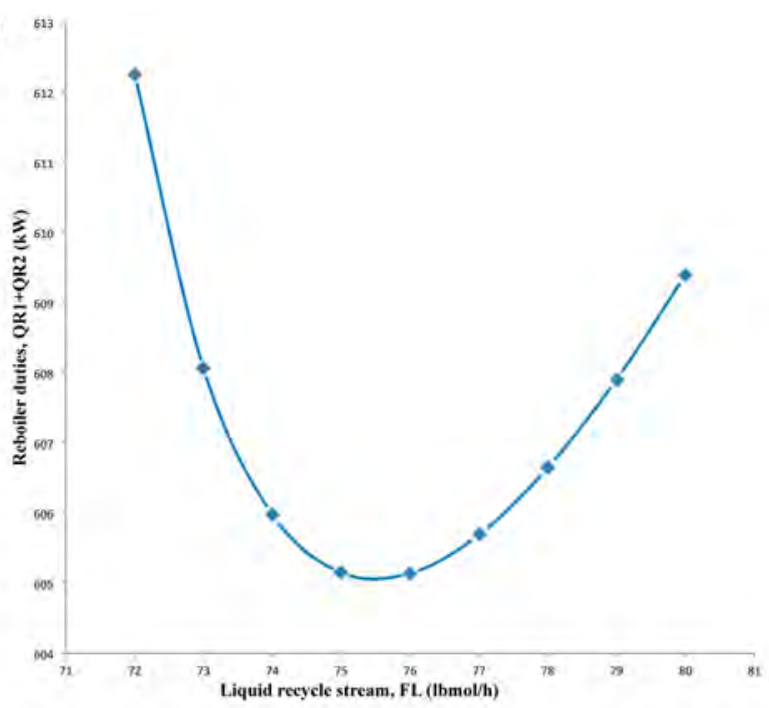

(b)

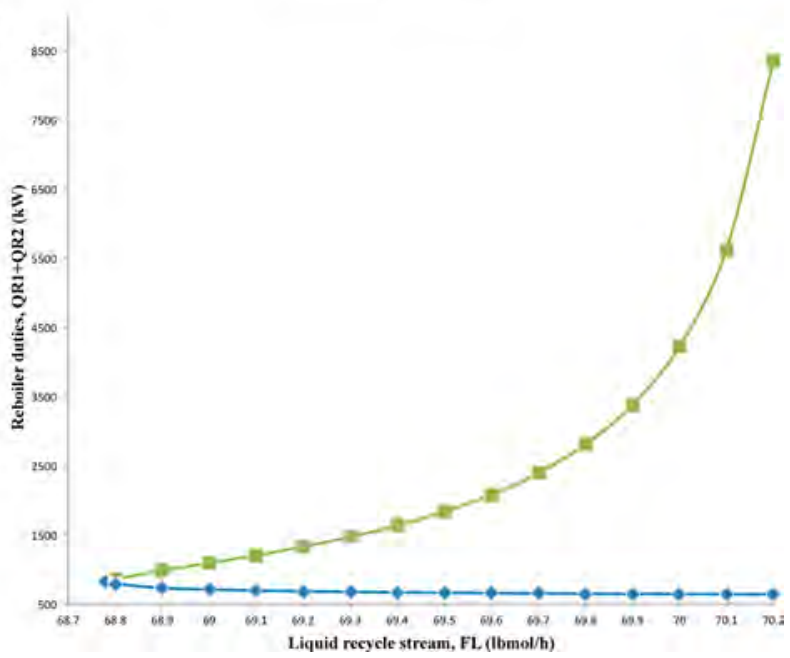

Figure 4. Energy optimization of the TCDS-I: (a) forward search (blue), (b) backward search (green).

matrices are suitable for SVD analysis using the complex transform $s=w j$.

Figures 5 and 6 provide the minimum singular value and condition number for two multiple steady states of TCDS-D. A small difference between the minimum singular values of the two multiple steady states can be observed for small frequencies (Figure 5). Furthermore, the condition number for the higher energy requirement steady state is slightly greater than that of the lower energy requirement steady state. It is important to note that small frequencies are related to steady state conditions. In general, the minimum singular values for the steady state with lower energy requirement are lower than those of the steady state with higher energy demand. As a result, the steady state with higher demand of energy is more controllable than that with lower energy demand.

In addition, condition numbers are higher for the steady state with lower energy. According to SVD results, the steady state with higher energy requirement is expected to be better conditioned with respect to the effect of disturbances.

When the TCDS-I was studied using SVD, similar results from those exhibited in TCDS-D were obtained. According to the results depicted in Figure 7, the sequence with higher energy requirement has higher minimum singular values than those of the sequence with lower energy consumption in a wide frequency range. In addition, according to Figure 8, the condition numbers for the higher energy requirement steady state are lower than those of the lower energy requirement state. As result, better control properties for the TCDS-I with higher energy demand can be expected.

Then, in general, the TCDS system with higher energy requirements exhibited better theoretical control properties than those of the same TCDS scheme with lower energy demand.

Notice that the theoretical control properties (minimum singular value and condition number) can be used to infer the closed-loop dynamic behavior of the thermally coupled distillation sequences; for that reason, only representative closed-loop dynamic simulations are needed. This is the main advantage of using the singular value decomposition technique.

To complement the controllability study, representative closed-loop dynamic responses were obtained for set point changes in the composition of the products and disturbances in the feed composition. The pairings in the feedback control loops were established according to industrial heuristics, that is, the composition of the distillate was achieved by manipulating the reflux ratio and the composition of the bottoms product was adjusted by manipulating the heat duty supplied to the reboiler. PI controllers were implemented and tuned by using the integral absolute of the error in the Aspen Dynamics simulator. As a representative case, Figure 9 contains two control loops implemented in the TCDS-D. Tuning parameters for the PI controllers for the lower energy requirement state were $K_{\mathrm{C}}=65.48$ and $\tau_{\mathrm{I}}=2.45 \mathrm{~min}$ for controller PIC5, and $K_{\mathrm{C}}$ $=25.58$ and $\tau_{\mathrm{I}}=12.85 \mathrm{~min}$ for controller PIC7. Tuning parameters for PI controllers for the higher energy requirement state were $K_{\mathrm{C}}=69.03, \tau_{\mathrm{I}}=2.44 \mathrm{~min}$ and $K_{\mathrm{C}}=101.58, \tau_{\mathrm{I}}=$ $0.851 \mathrm{~min}$ for controllers PIC5 and PIC7, respectively.

Taking the TCDS-D as our case study, Figure 10 shows the dynamic responses of the compositions of pentane and heptane when positive set point changes of 0.01 are implemented. Figure 10a for the composition of $\mathrm{C} 5$ indicates that, in both cases, the set point changes are achieved properly, but in the control loop of $\mathrm{C} 7$, the state with higher demand of energy achieves the set point faster (Figure 10b).

Further disturbances in feed stream compositions of $+5 \%$ in $\mathrm{C} 5,-2.5 \%$ in C6, and $-2.5 \%$ in C7 were introduced. Figure 11 shows the dynamic closed-loop responses for pentane and

Table 3. Design Variables for the TCDS-D for Two States Using FV $=140 \mathrm{lb}-\mathrm{mol} / \mathrm{h}$

\begin{tabular}{lccccc} 
& \multicolumn{2}{c}{ state with low energy requirement } & & \multicolumn{2}{c}{ state with high energy requirement } \\
\cline { 2 - 3 } \cline { 5 - 6 } \multicolumn{1}{c}{ variable } & column C-1 & column C-2 & 2.64 & column C-1 & column C-2 \\
diameter (ft) & 2.03 & 1.90 & 2.00 & 4.40 \\
reflux ratio $(\mathrm{RR})$ & 2.42 & 0 & 665.32 & 2.34 & 50 \\
reboiler duty $\mathrm{Q}(\mathrm{kW})$ & & & 0 & 4087.78
\end{tabular}


Table 4. Design Variables for the TCDS-I for Two States Using FL $=69.5 \mathrm{lb}-\mathrm{mol} / \mathrm{h}$

\begin{tabular}{|c|c|c|c|c|}
\hline \multirow[b]{2}{*}{ variable } & \multicolumn{2}{|c|}{ state with low energy requirement } & \multicolumn{2}{|c|}{ state with high energy requirement } \\
\hline & column C-1 & column C-2 & column C-1 & column $\mathrm{C}-2$ \\
\hline diameter $(\mathrm{ft})$ & 2.12 & 3.57 & 2.13 & 4.03 \\
\hline reflux ratio ( $R R$ ) & 0 & 3.06 & 0 & 77.8 \\
\hline reboiler duty Q (kW) & 432.50 & 132.09 & 413.59 & 10170.00 \\
\hline
\end{tabular}

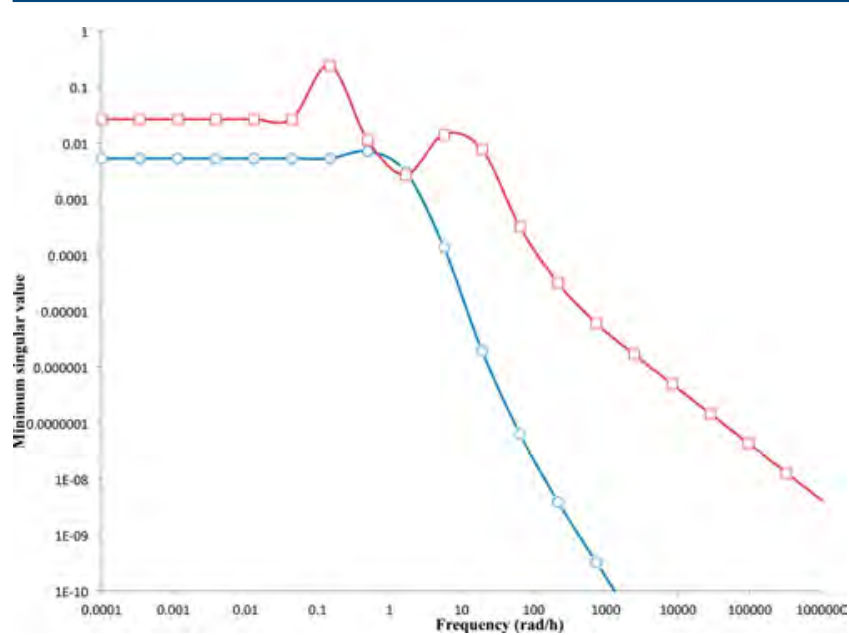

Figure 5. Minimum singular values for TDCS-D. Lower energy requirement steady state $(O)$ and higher energy requirement steady state $(\square)$.

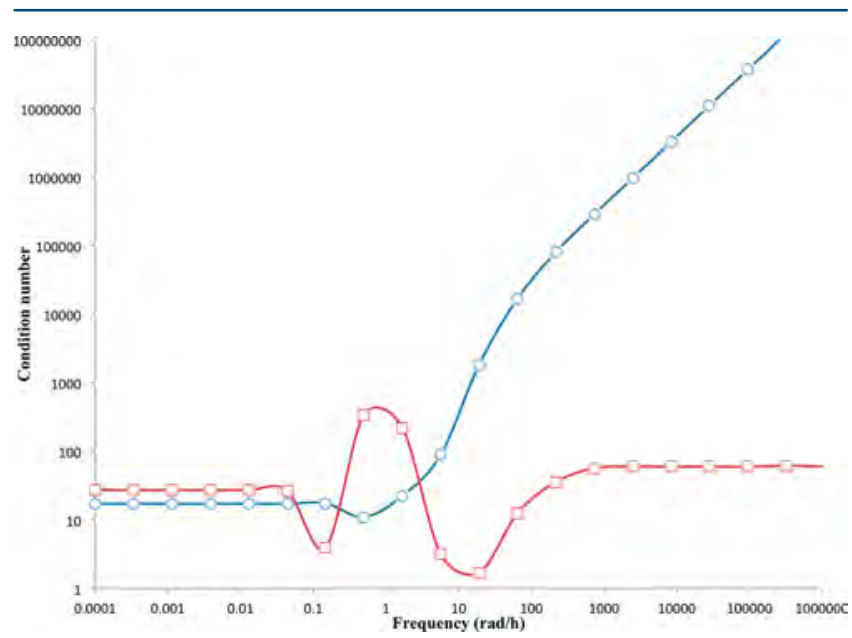

Figure 6. Condition numbers for TCDS-D. Lower energy requirement steady state $(O)$ and higher energy requirement steady state $(\square)$.

heptane compositions. The steady state with higher energy requirement shows an appropriate control performance because the disturbances introduced in the feeding stream can be eliminated in the composition of the distillate C5. Figure $11 \mathrm{~b}$ shows that the dynamic response of $\mathrm{C} 7$ does not have a significant deviation from the nominal point. On the contrary, the composition of $\mathrm{C} 7$ in the state with the lower energy requirement presents important oscillations before adjusting the composition. Similar trends were observed in the closedloop dynamic responses obtained in the TCDS-I.

In general and according to the closed-loop dynamic responses, both states can achieve changes in set points and eliminate the effect of some input disturbances, therefore it can be inferred that both states can be controlled using PI

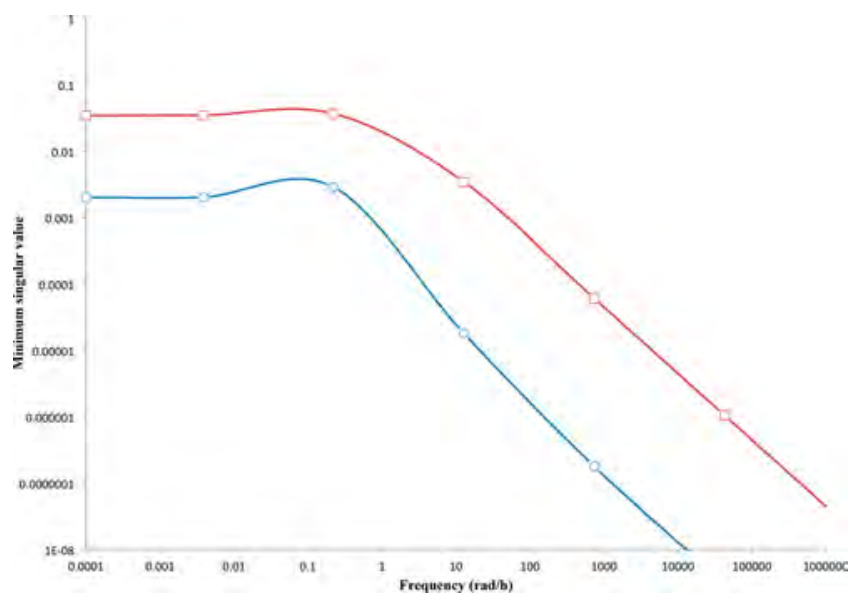

Figure 7. Minimum singular values for TDCS-I. Lower energy requirement steady state $(\mathrm{O})$ and higher energy requirement steady state $(\square)$.

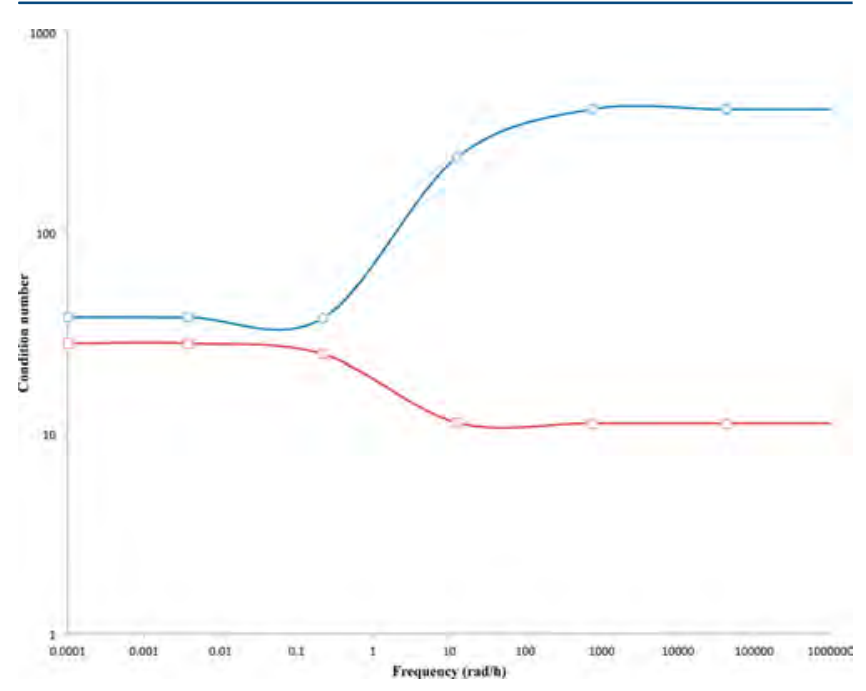

Figure 8. Condition numbers for TCDS-I. Lower energy requirement steady state $(O)$ and higher energy requirement steady state $(\square)$.

controller, but the control is favored in the state of high energy demand.

\section{CONCLUSIONS}

Thermally coupled distillation sequences with side columns have been designed and optimized for minimum energy consumption, and the results indicate that multiple steady state solutions can occur. As a result, during energy optimization it is important to detect all operational regions, since they may differ significantly in energy consumption. This is significant since, in industrial practice, it is convenient to select the design with the minimum energy consumption in order to cut operational and capital costs. 


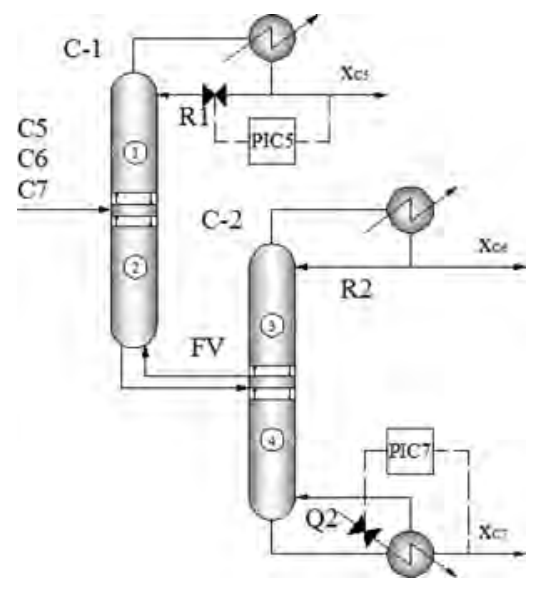

Figure 9. Representative control loops in the TCDS-D.
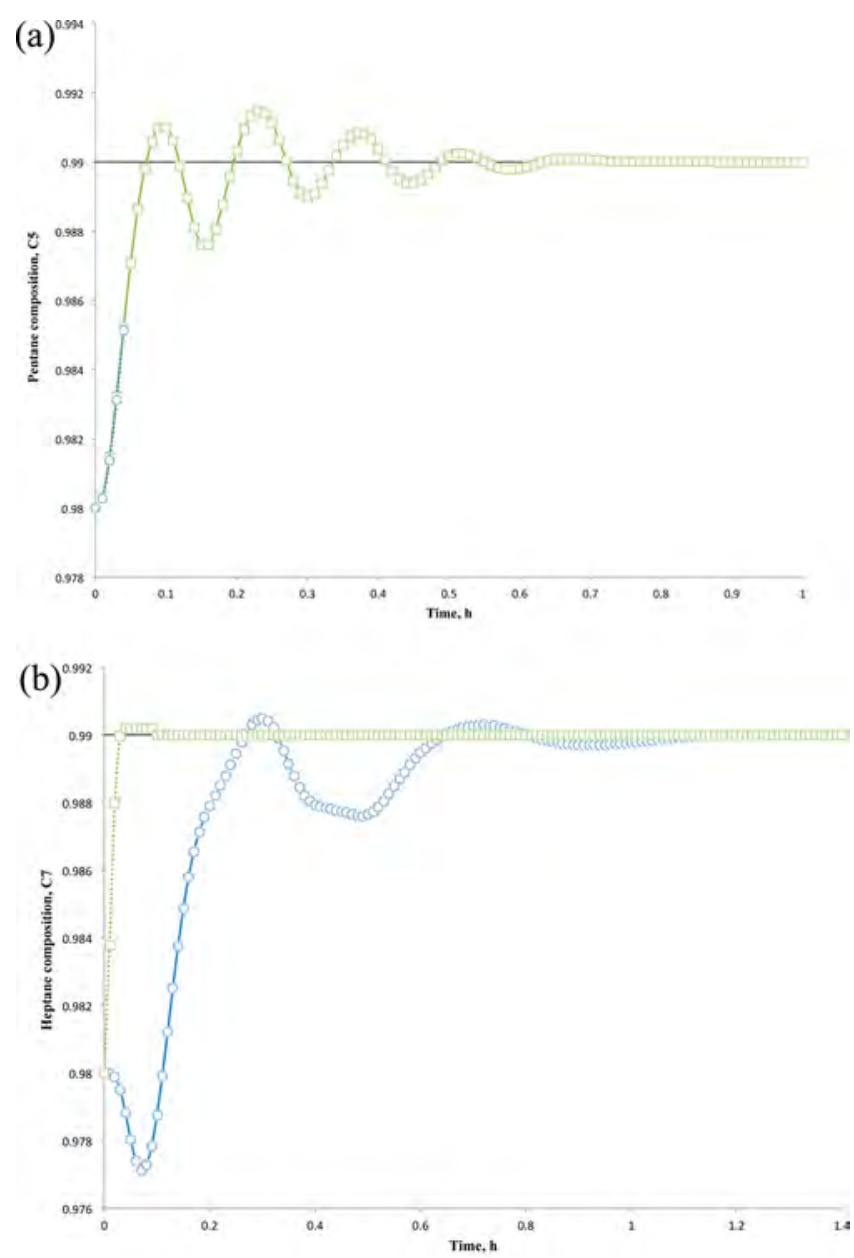

Figure 10. Dynamic responses for set point changes of +0.01 for pentane and heptane compositions in TCDS-D: (a) pentane, (b) heptane. Lower energy requirement steady state $(O)$, higher energy requirement steady state $(\square)$.

Regarding controllability issues, the state with higher energy requirements showed better control properties than those of sequences with lower energy requirements. However, both steady states can be controllable using PI controllers. As the main conclusion, it is possible to operate the thermally coupled distillation sequences under minimum energy consumption at expenses of a more tight control. Nevertheless, this is an initial
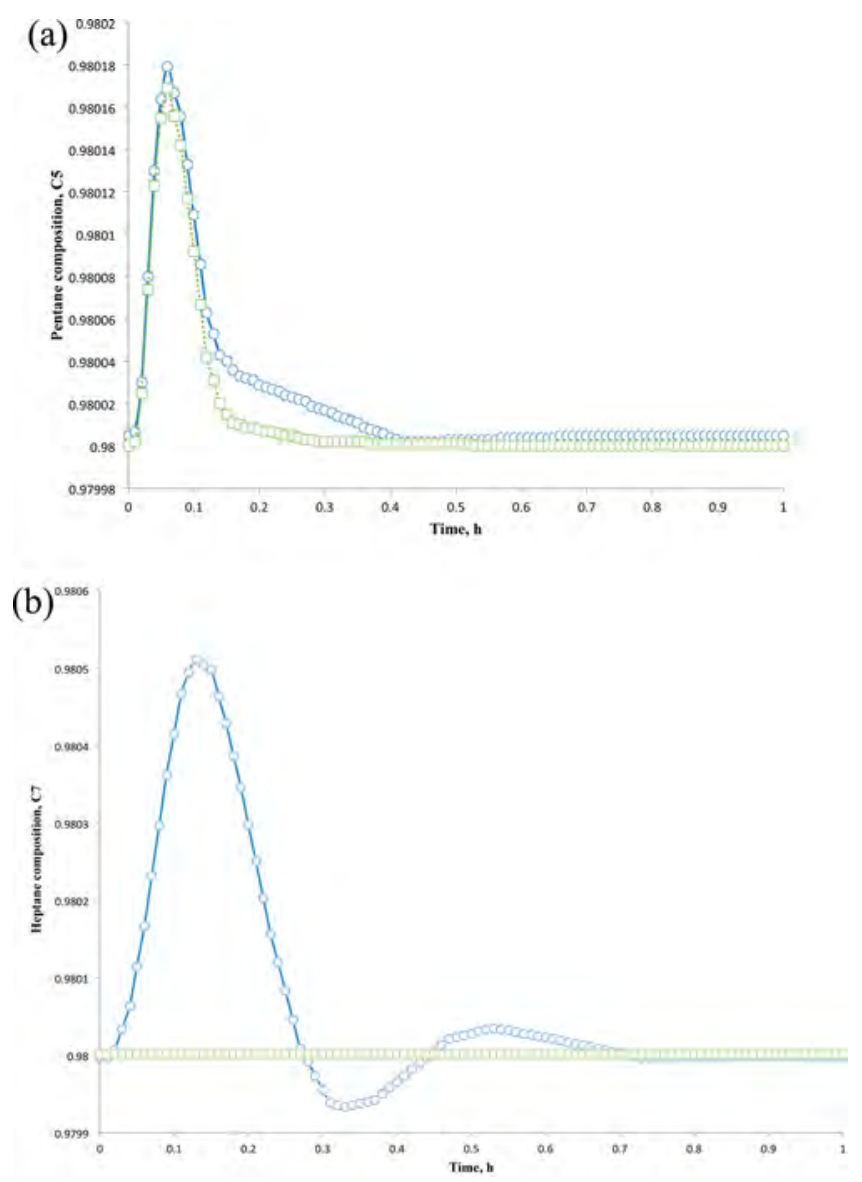

Figure 11. Dynamic responses for perturbations on feed composition in TCDS-D. (a) pentane, (b) heptane. Lower energy requirement steady state $(O)$, higher energy requirement steady state $(\square)$.

study and more cases of study should be analyzed to extend the scope of our conclusion; for instance, nonideal mixtures or reactive systems should be considered in those complementary studies.

\section{ASSOCIATED CONTENT}

S Supporting Information

Tables presenting the transfer matrices for the two steady states of the thermally coupled distillation sequences. This material is available free of charge via the Internet at http://pubs.acs.org.

\section{AUTHOR INFORMATION}

\section{Corresponding Author}

*Tel.: +524737320006 ext 8142. Fax.: +524737320006 ext 8139. E-mail: hernasa@ugto.mx.

Notes

The authors declare no competing financial interest.

\section{ACKNOWLEDGMENTS}

The authors acknowledge the financial support provided by CONACYT (Mexico).

\section{REFERENCES}

(1) Tedder, D. W.; Rudd, D. F. Parametric Studies in Industrial Distillation: Part I. Design Comparisons. AIChE J. 1978, 24, 303.

(2) Flores, O. A.; Cárdenas, J. C.; Hernández, S.; Rico-Ramírez, V. Thermodynamic Analysis of Thermally Coupled Distillation Sequences. Ind. Eng. Chem. Res. 2003, 42, 5940. 
(3) Glinos, K.; Malone, F. Optimality Regions for Complex Column Alternatives in Distillation Systems. Chem. Eng. Res. Des. 1988, 66, 229.

(4) Hernández, S.; Jímenez, A. Design of Optimal ThermallyCoupled Distillation Systems Using a Dynamic Model. Trans IChemE 1996, 74, 357.

(5) Hernández, S.; Jímenez, A. Controllability Analysis of Thermally Coupled Distillation Systems. Ind. Eng. Chem. Res. 1999a, 38, 3957.

(6) Triantafyllou, C.; Smith, R. The Design and Optimization of Fully Thermally Coupled Distillation Columns. Trans Inst. Chem. Eng. 1992, 70, 118.

(7) Rong, B. G.; Kraslawski, A.; Turunen, I. Synthesis of Functionally Distinct Thermally Coupled Configurations for Quaternary Distillation. Ind. Eng. Chem. Res. 2003, 42, 1204.

(8) Wolff, E. A.; Skogestad, S. Operation of Integrated ThreeProduct (Petlyuk) Distillation Columns. Ind. Eng. Chem. Res. 1995, 34, 2094.

(9) Hernández, S.; Jímenez, A. Design of Energy-Efficient Petlyuk Systems. Comput. Chem. Eng. 1999b, 23, 1005.

(10) Segovia-Hernández, J. G.; Hernández, S.; Rico-Ramírez, V.; Jiménez, A. A Comparison of the Feedback Control Behavior between Thermally Coupled and Conventional Distillation Schemes. Comput. Chem. Eng. 2004, 28, 811.

(11) Gutiérrez-Guerra, R.; Segovia-Hernández, J. G.; Hernández, S. Reducing Energy Consumption and $\mathrm{CO}_{2}$ Emissions in Extractive Distillation. Chem. Eng. Res. Des. 2009, 87, 145.

(12) Cárdenas-Guerra, J. C.; López-Arenas, T.; Lobo-Oehmichen, R.; Pérez-Cisneros, E. S. A Reactive Distillation Process for Deep Hydrodesulfurization of Diesel: Multiplicity and Operation Aspects. Comput. Chem. Eng. 2010, 34, 196.

(13) Kano, M.; Makita, H.; Hasebe, S. Prediction of Multiple Steady States in Distillation through Simple Mass and Heat Balance Analysis. Ind. Eng. Chem. Res. 2011, 50, 1346.

(14) Purohit, L. J.; Mahajani, M. S.; Patwardhan, C. S. Analysis of Steady-State Multiplicity in Reactive Distillation Columns. Ind. Eng. Chem. Res. 2013, 52, 5191.

(15) Dejanović, I.; Matijašević, L.; Olujić, Ž. Dividing Wall Column-A Breakthrough Towards Sustainable Distilling. Chem. Eng. Process. 2010, 49, 559.

(16) Asprion, N.; Kaibel, G. Dividing Wall Columns: Fundamentals and Recent Advances. Chem. Eng. Process. 2010, 49, 39.

(17) Yildirim, Ö.; Kiss, A. A.; Kenig, E. Y. Dividing Wall Columns in Chemical Process Industry: A Review on Current Activities. Pur. Technol. 2011, 80, 403.

(18) Delgado-Delgado, R.; Hernández, S.; Barroso-Muñoz, O. F.; Segovia-Hernández, J. G.; Castro-Montoya, A. J. From Simulation Studies to Experimental Tests in a Reactive Dividing Wall Distillation Column. Chem. Eng. Res. Des. 2012, 90, 855.

(19) Stakka, D.; Grütznera, T.; Schweglera, B.; Roedererb, D. Dividing Wall Column for Industrial Multipurpose Use. Chem. Eng. Process. 2014, 75, 48.

(20) Delgado-Delgado, R.; Hernández, S.; Barroso-Muñoz, O. F.; Segovia-Hernández, J. G.; Rico-Ramírez, V. Some Operational Aspects and Applications of Dividing Wall Columns: Energy Requirements and Carbon Dioxide Emissions. Clean. Technol. Envir. 2014, in press.

(21) Gani, R.; Jorgensen, S. B. Multiplicity in Numerical Solution of Non-linear Models: Separation Processes. Comput. Chem. Eng. 1994, $18,55$. 